PASJ: Publ. Astron. Soc. Japan , 1-??,

(C) 2021. Astronomical Society of Japan.

\title{
Panoramic Views of Cluster-Scale Assemblies Explored by Subaru Wide-Field Imaging *
}

\author{
Tadayuki KodAmA ${ }^{1}$, \\ Masayuki TAnaka ${ }^{2}$, Takayuki Tamura ${ }^{10}$, Hideki Yahagi ${ }^{1}$, Masahiro Nagashima ${ }^{7}$, Ichi TAnakA ${ }^{5}$, \\ Nobuo Arimoto ${ }^{1}$, Toshifumi Futamase ${ }^{5}$, Masanori IYe ${ }^{1}$, Yoshikazu Karasawa ${ }^{5}$, \\ Nobunari Kashikawa ${ }^{1}$, Wataru Kawasaki ${ }^{9}$, Tetsu KitaYama ${ }^{11}$, Hideo Matsuhara ${ }^{10}$, \\ Fumiaki NakAta $^{8}$, Takaya Ohashi ${ }^{12}$, Kouji Oнta ${ }^{6}$, Takashi Oкамото ${ }^{1,8}$, \\ Sadanori OKamura ${ }^{2,4}$, Kazuhiro Shimasaku ${ }^{2,4}$, Yasushi Suto $^{3,4}$, Naoyuki Tamura $^{8}$, \\ Keiichi UMETsu $^{9}$, and Toru YAMADA ${ }^{1}$ \\ ${ }^{1}$ National Astronomical Observatory of Japan, Mitaka, Tokyo 181-8588, Japan \\ kodama@optik.mtk.nao.ac.jp \\ ${ }^{2}$ Department of Astronomy, School of Science, University of Tokyo, Tokyo 113-0033 \\ ${ }^{3}$ Department of Physics, School of Science, University of Tokyo, Tokyo 113-0033 \\ ${ }^{4}$ Research Center for the Early Universe, School of Science, University of Tokyo, Tokyo 113-0033 \\ ${ }^{5}$ Astronomical Institute, Tohoku University, Aoba-ku, Sendai 980-8578 \\ ${ }^{6}$ Department of Astronomy, Kyoto University, Sakyo-ku, Kyoto 606-8502 \\ ${ }^{7}$ Department of Physics, Kyoto University, Sakyo-ku, Kyoto 606-8502 \\ ${ }^{8}$ Department of Physics, University of Durham, South Road, Durham DH1 3LE, UK \\ ${ }^{9}$ Institute of Astronomy and Astrophysics, Academia Sinica, Taipei 106, Taiwan \\ ${ }^{10}$ Institute of Space and Astronautical Science, Sagamihara, Kanagawa 229-8510 \\ ${ }^{11}$ Department of Physics, Toho University, Funabashi, Chiba 274-8510 \\ ${ }^{12}$ Department of Physics, Tokyo Metropolitan University, Hachioji, Tokyo 192-0397
}

(Received 2004 December 4; accepted 2005 February 21)

\begin{abstract}
We have started PISCES project; a panoramic imaging and spectroscopic survey of distant clusters on Subaru. It exploits the unique wide-field imaging capability of Suprime-Cam, which provides a $34^{\prime} \times 27^{\prime}$ field of view corresponding to a physical area of $16 \times 13 \mathrm{Mpc}^{2}$ at $z \sim 1$. We plan to target 15 clusters in total at $0.4 \lesssim z \lesssim 1.3$. In this paper, we report on our first results concerning the inner structures and largescale structures of two distant clusters at $z=0.55$ and 0.83 together with the earlier results on a $z=0.41$ cluster. The photometric redshift technique has been applied to multi-color data in order to efficiently remove most of the foreground/background galaxies so as to isolate the cluster member candidates. We have found large-scale filamentary structures around the clusters, extending out to $>5 \mathrm{Mpc}$ from the cores, as well as complex inner structures. The galaxy distributions in the inner regions of the clusters look similar to the X-ray intensity maps, suggesting that most of the optical structures trace physically bound systems. We also compared the structures of the three clusters with those of model clusters in a numerical simulation $(N$-body + semi-analytic model $)$ by parameterising the shapes of the iso-density contours of galaxies, and found a broad agreement. Our results provide good evidence that cluster-scale assembly takes place along filaments during hierarchical clustering, while the structures found here need to be confirmed spectroscopically in the near future.

Key words: galaxies: clusters - galaxies: clusters: individual (CL 0939+4713, CL 0016+1609, RX J0152.7-1357 — galaxies: evolution
\end{abstract}

\section{Introduction}

Clusters of galaxies are the largest-scale objects in the Universe in which dynamical relaxation has fairly advanced. They consist of 100-1000 galaxies, X-ray emitting hot plasma, and dark matter, which binds the systems.

\footnotetext{
Based in part on data collected at the Subaru Telescope, which is operated by the National Astronomical Observatory of Japan. Based also in part on observations obtained with XMM-Newton, an ESA science mission with instruments and contributions directly funded by ESA Member States and NASA, USA.
}

Since the dynamical time scale of clusters is comparable to the Hubble time, these systems are still presently dynamically evolving, and thus are supposed to somehow keep memory of the cosmological initial conditions. For this reason, clusters of galaxies are often used as cosmological probes at various wavelengths (e.g., Kitayama et al. 1998). Now that the cosmological parameters have been determined with a reasonable accuracy by microwave background radiation and distant supernovae (Spergel et al. 2003), our primary motivation is now directed to understanding the major constituents of the Universe, such 
as galaxies, baryonic gas, and dark matter, which are playing major roles in clusters of galaxies.

According to the cold dark-matter scenario, small-scale objects collapsed first as a result of gravitational instability, and then they pulled together and merged one another to form bigger and bigger systems (dark-matter halos) with time. In each dark-matter halo the gas condensed and formed stars - the birth of shining galaxies. During the course of assembly to the larger systems (groups/clusters), galaxies experienced environmental effects by interactions with other galaxies, cluster potential, and/or intra-cluster gas. Hence, their star-formation activities as well as morphologies are largely affected and truncated (e.g., Larson et al. 1980; Lavery, Henry 1986; Moore et al. 1996; Abadi et al. 1999; Hashimoto, Oemler 2000). In this way, galaxy evolution must be closely related to the structure evolution of the Universe and the change of galaxy environment. These environmental effects (a posteriori) are very important because the well-known morphology-density relation (Dressler 1980; Dressler et al. 1997) and the origin of the Hubble sequence of galaxies are possibly related to these processes. These are additional effects on top of the intrinsic effects (a priori) set by the cosmological initial conditions, i.e., cluster cores started off from higher density peaks in the early Universe, where galaxies formed quickly in a biased manner. (e.g., Kaiser 1984; Bardeen et al. 1986; Cen, Ostriker 1993).

In spite of recent vigorous progress in this field, the central physical processes behind the environmental effects still remain elusive. The key possible processes that have been proposed include: (1) ram-pressure stripping, a sudden removal of gas by the interactions with the intracluster medium (ICM) (e.g., Abadi et al. 1999); (2) tidal encounters, often accompanied by an intense star burst (e.g., Lavery, Henry 1986; Hashimoto, Oemler 2000); and (3) strangulation, a gradual consumption of the remaining disk gas without any further gas supply from the halo (Larson et al. 1980). Being limited by the light-collection power of telescopes and/or narrow field coverage of detectors, observers have concentrated on either cluster cores or general fields, and have had little knowledge concering the transition regions that bridge these two extreme environments. We do not know the matter distribution (galaxy/dark-matter) there, either.

\subsection{PISCES Project}

\subsubsection{Basic concepts}

The advent of the Subaru Telescope (Iye et al. 2004) with a unique combination of great light-collection power and large field of view, has opened a new window in the study of distant clusters. This has enabled us to look into a more distant Universe $(z \sim 1)$ which has abundant information on galaxy/cluster evolution, and to reach fainter objects $\left(>M^{*}+1.5\right)$ where evolution is stronger. At the same time, with its wide-field camera Suprime-Cam (Miyazaki et al. 2002) covering 30' (14 Mpc in physical scale or $29 \mathrm{Mpc}$ in comoving scale at $z=1$ ), we can view from cluster cores through the tran- sition region out to the general field, all at once. Taking this unique advantage of Subaru, we started the PISCES project (Panoramic Imaging and Spectroscopy of Cluster Evolution with Subaru) by combining several active research groups in Japan working on clusters of galaxies in various aspects (optical-NIR/X-ray/lensing/simulation). This large collaborative project aims to carry out systematic deep and wide observations of distant clusters at various evolutionary stages, and compare their fundamental physical quantities (mass, luminosity, colors, spectral indices, morphology, and kinematics) in detail as a function of the environment (structure, local density) and time (redshift). This process will eventually prove when, where and how the galaxies form and evolve. The basic idea is to utilize clusters of galaxies as landmarks in the distant Universe, and to map out the large-scale structures around them, and reconstruct the history of galaxy evolution along with the hierarchical structure formation of the Universe. The critical issues we aim to address with PISCES are the following two:

- Cluster-scale assembly and the spatial bias: We will map out the large-scale structure beyond the cluster cores (e.g., West et al. 1998; Lubin et al. 2000) and trace the history of cluster-scale assembly along the filaments. By using the weak-lensing technique, we will also map the dark-matter distribution. Galaxies do not necessarily trace the distribution of dark matter faithfully (e.g., Kaiser 1984; Bardeen et al. 1986; Cen, Ostriker 1993). By quantifying this galaxy formation bias as a function of the redshift, scale and density, we will place strong constraints on the galaxy formation model.

- Star formation history and the environmental effect: By comparing the galaxy properties as a function of environment and time with the aid of a population synthesis technique, we will derive the 'environment-dependent' star-formation rate, mass, and morphology of galaxies. By watching when, where, and how the environmental effects exert on galaxies, we will identify the major physical processes involved in morphological transformation, and reveal the origin of the Hubble sequence of galaxies.

\subsubsection{Cluster sample}

The total planned targets of our PISCES project are $15 \mathrm{X}$-ray detected clusters at $0.4<z<1.3$, covering various evolutionary stages $(z)$ and richness $\left(L_{\mathrm{X}}\right)$, in coordination with XMM/Chandra and HST/ACS observations (see table 1).

The criteria of our target selection are the following:

- X-ray detection: We select clusters that are detected in X-rays. X-ray selection is less prone to the projection effect along the line of sight than optical selection. Although we may be biased towards the densest regions at all epochs, this is not very problematic in a time-sequence comparison, since the wide-field imaging will cover all environments from the dens- 
est cluster cores to the nearby groups and to the low-density filaments connecting the clumps.

- Redshift range: We limit the cluster sample to the redshift range of $0.4 \lesssim z \lesssim 1.3$, so that the SuprimeCam filters alone in the optical $\left(B V R i^{\prime} z^{\prime}\right)$ can neatly cover the $4000 \AA$ break feature in the rest-frame, and hence the photometric redshift technique works well (subsection 2.2).

- Redundancy: We select multiple (3-5) clusters in each of the four redshift ranges $(z \sim 0.4,0.55,0.85$, and 1.2). Clusters of galaxies are statistical objects by nature, and are likely to have large varieties; it is thus important to average the properties over many clusters of different richness at a given epoch to obtain general views.

- Coordination with other projects: We include many common targets with other major cluster projects, especially with XMM/Chandra and HST/ACS observations (e.g., Blakeslee 2001, private communication). Such coordination is important, since diffuse X-ray emission traces the distribution of gravitationally bound systems and provides the properties of hot intra-cluster media, such as temperature and chemical composition. HST/ACS gives us deep images of high spatial resolution, and hence provides essential morphological information of galaxies. Our sample also includes a cluster at the NEP region where the visibility of ASTRO-F (Japanese Space NIR-FIR mission, providing $10^{\prime} \times 10^{\prime}$ fieldof-view; Shibai 2003; Murakami 2005) is good; we plan for a follow-up observation to search for dusty star-burst phenomena and to improve our photometric redshifts. Also note that all of our clusters above $z=0.6$ are targets for space-IR imaging by the Spitzer Telescope. We also include seven clusters that have existing detailed Sunyaev-Zeldovich effect (S-Z) maps for comparing of the sub-structures in the cluster cores.

- Visibility: The maximum elevation must be $>$ $40^{\circ}$ (airmass < 1.6) from the Subaru Telescope at Mauna Kea in Hawaii for efficient observations.

Note that our total sample includes some GTO (guaranteed time observations) targets of the Suprime-Cam team (CL 1604 and RX J0849) and those targeted by different teams, including some of our members (CL 0024, CL 0939, MS 0451, and MS1054). These are appended to the PISCES targets, since the imaging data of these distant clusters are taken under similar scientific motivations, and the data are useful if viewed under the same context and in a systematic way.

\subsection{This Paper}

In 2003 September, as a part of the PISCES project, we successfully obtained complete imaging data-set for the two clusters CL 0016+1609 ( $z=0.55$; hereafter CL 0016) and RX J0152.7-1357 ( $z=0.83$; hereafter RX J0153) In this paper, we concentrate on three clusters in total; the above newly observed two clusters CL 0016, RX J0153, and a previous target CL $0939+4713(z=0.41$; hereafter CL 0939) for comparison (Kodama et al. 2001). These three clusters under investigation are located in a sequence of redshifts corresponding to lookback times of 4.3, 5.4, and 7.0 Gyrs, and are therefore suited for us to look for possible signatures of evolutionary effects in clusters.

The first results on the CL $0939+4713$ cluster were already reported by Kodama et al. (2001), showing largescale filamentary structures around this cluster and a transition of galaxy colors in the group environment along the filaments. Note that Iye et al. (2000) also presented their Subaru/Suprime-Cam image of CL 0939+4713 taken in their GT phase. This paper expands Kodama et al.'s (2001) analysis (for CL 0939+4713) to two additional higher redshift clusters, concentrating on the large-scale structures. The environmental dependence of the photometric properties of galaxies in these two clusters and those in local counterparts from SDSS (Sloan Digital Sky Survey; York et al. 2000) will be presented in Tanaka et al. (2005).

We adopt the cosmological parameters $\left(h_{70}, \Omega_{m}\right.$, $\left.\Omega_{\Lambda}\right)=(1.0,0.3,0.7)$ throughout this paper, where $h_{70}$ is defined as $H_{0} /\left(70 \mathrm{~km} \mathrm{~s}^{-1} \mathrm{Mpc}^{-1}\right)$. With this parameter set, $1^{\prime}$ corresponds to physical scales of $0.33,0.38$, and $0.46 \mathrm{Mpc}$, or comoving scales of $0.46,0.59$, and $0.84 \mathrm{Mpc}$, at cluster redshifts of $0.41,0.55$, and 0.83 , respectively. All of the magnitudes used in this paper will be given in the AB-magnitude system.

\section{Observations, Reduction, and Analysis}

\subsection{Observations and Reduction}

Imaging data of CL 0016 and RX J0153 were obtained with Suprime-Cam (0.'202 per pixel, and $34^{\prime} \times$ $27^{\prime}$ field of view) mounted on the Subaru Telescope at Mauna Kea during the nights of 2003 September 25-26. Images were obtained through several broad-band filters ( $B V R i^{\prime} z^{\prime}$ and $V R i^{\prime} z^{\prime}$, respectively). The net exposure times and the 5- $\sigma$ limiting magnitudes are summarized in table 2. The seeing was extremely stable during these nights and across the bands, and its sizes were always between $0 .^{\prime \prime} 5$ and 0.'65 (FWHM). Therefore, our combined frames achieved the uniform seeing size of $0 . " 65$ in all passbands for both clusters. The sky conditions were photometric, and the photometric zero-points were calibrated based on the Landolt (1992) standard stars in $B, V$, and $R$-bands, and $i^{\prime}$ and $z^{\prime}$-band images were calibrated directly onto the SDSS system (Fukugita et al. 1995) based on the spectro-photmetric standard stars. After correcting for the Galactic extinction (Schlegel et al. 1998), we checked the accuracy of our photometric zero-points. We compared the colors of Galactic stars in our field with those of Gunn and Stryker (1983) stars (stellar SEDs convolved with Suprime-Cam responses). It turned out that our colors are slightly shifted with respect to the GS stars ( $\sim 0.1$ magnitude at most). Although we do not know the exact reason for this discrepancy, it may be partly because the filter response functions, including CCD sensitivity curves, are slightly different between SDSS and 
Table 1. Total PISCES targets (planned).

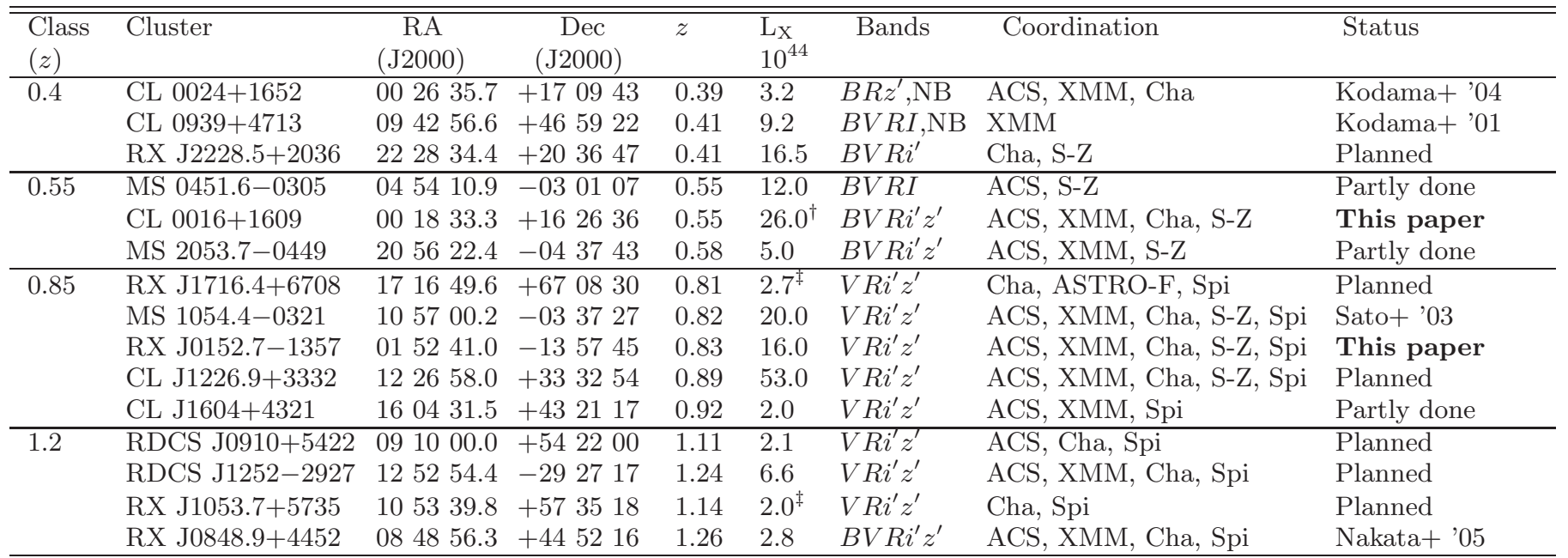

$\mathrm{L}_{\mathrm{X}}$ shows the bolometric X-ray luminosity in units of $10^{44} \mathrm{erg} \mathrm{s}^{-1}\left(H_{0}=70\right)$ with some exceptions $\left({ }^{\dagger} 0.4-10 \mathrm{kev},{ }^{\ddagger} 0.5-2 \mathrm{kev}\right)$. 'NB' in the 7th column indicates narrow-band imaging to target $\mathrm{H} \alpha$ line at the cluster redshift. In the 8th column, 'XMM' and 'Cha' indicate the XMM and Chandra targets, respectively. 'ACS' indicates the HST/ACS targets and 'S-Z' indicates the Sunyaev-Zeldovich effect targets in Radio. 'ASTRO-F' and 'Spi' indicate the targets for ASTRO-F and Spitzer space-IR telescopes, respectively.

Refs. for $L_{X}$ : CL 0024 (Ota et al. 2003); CL 0939 (De Filippis et al. 2003); RX J2228 (Pointecouteau et al. 2002), MS 0451 (Donahue \& Stocke 1995); CL 0016 (Worrall \& Birkinshaw 2003); MS 2054 (Henry 2000); RX J1716 (Henry et al. 1997); MS 1054 (Jeltema et al. 2001); RX J0153 (Maughan et al. 2003); RX J1227 (Maughan et al. 2004); CL 1604 (Lubin et al. 2004); RDCS J0910 (Stanford et al. 2002); CL 1252 (Rosati et al. 2004); RX J1054 (Hasinger et al. 1998); and RX J0849 (Stanford et al. 2001).

Refs. for S-Z: MS 1054, RX J0153, RX J1227 (Joy et al. 2001); MS 0451, CL 0016, MS 2054, MS 1054 (Grego et al. 2001 ); RX J2228 (Pointecouteau et al. 2002).

Table 2. Summary of the observations.*

\begin{tabular}{lccccc}
\hline \hline Cluster & Passband & Date & $\begin{array}{c}\text { Exp. times } \\
(\text { total })\end{array}$ & $\begin{array}{c}\text { Lim. mag. } \\
(5 \sigma)\end{array}$ & $\begin{array}{c}\text { Seeing } \\
(\mathrm{FWHM})\end{array}$ \\
\hline CL 0939+4713 & $B$ & 2001 Jan 21-22 & $60 \mathrm{~min}$ & 26.6 & $\sim 1 !^{\prime \prime} 1$ \\
& $V$ & 2001 Jan 21-22 & $36 \mathrm{~min}$ & 26.0 & $\sim 0 !^{\prime \prime} 7$ \\
& $R$ & 2001 Jan 21-22 & $66 \mathrm{~min}$ & 26.0 & $\sim 1 .^{\prime \prime} 0$ \\
& $I$ & 2001 Jan 21-22 & $21 \mathrm{~min}$ & 25.5 & $\sim 0 !^{\prime \prime} 7$ \\
\hline CL 0016+1609 & $B$ & 2003 Sep 25-26 & $90 \mathrm{~min}$ & 26.9 & $\sim 0 !^{\prime \prime} 65$ \\
& $V$ & 2003 Sep 25-26 & $96 \mathrm{~min}$ & 26.2 & $\sim 0 !^{\prime \prime} 65$ \\
& $R$ & 2003 Sep 25-26 & $64 \mathrm{~min}$ & 26.0 & $\sim 0 !^{\prime \prime} 65$ \\
& $i^{\prime}$ & 2003 Sep 25-26 & $60 \mathrm{~min}$ & 25.9 & $\sim 0 !^{\prime \prime} 65$ \\
& $z^{\prime}$ & 2003 Sep 25-26 & $47.5 \mathrm{~min}$ & 24.6 & $\sim 0 !^{\prime \prime} 65$ \\
\hline RX J0152.7-1357 & $V$ & 2003 Sep 25-26, 2003 & $120 \mathrm{~min}$ & 26.7 & $\sim 0 !^{\prime \prime} 65$ \\
& $R$ & 2003 Sep 25-26, 2003 & $116 \mathrm{~min}$ & 26.5 & $\sim 0 !^{\prime \prime} 65$ \\
& $i^{\prime}$ & 2003 Sep 25-26, 2003 & $75 \mathrm{~min}$ & 26.1 & $\sim 0 !^{\prime \prime} 65$ \\
& $z^{\prime}$ & 2003 Sep 25-26, 2003 & $77 \mathrm{~min}$ & 25.0 & $\sim 0 .^{\prime \prime} 65$ \\
\hline
\end{tabular}

* Limiting magnitudes are measured within $2^{\prime \prime}$ apertures. Seeing sizes are measured from the combined frames. 
our observations with Suprime-Cam. We shifted the zeropoints of the photometric catalogues so as to match with the GS star colors in the same manner as in Ajiki et al. (2003).

The data were reduced with the IRAF and NEKOSOFT (Yagi et al. 2002) software packages, following standard procedures of bias subtraction and flatfielding. The latter was achieved using supersky (self) flats constructed from the median of the dithered science frames (objects are masked). We then combined the mosaiced chips, carefully matching the relative flux among them. Note that, since the seeing was very stable during the nights, we performed no point-spread function (PSF) equalization in order to avoid image degradation. Color pictures of the central regions $\left(3^{\prime} \times 3^{\prime}\right)$ of the two clusters are shown in figures 1 and 2 .

Galaxies are detected in the $i^{\prime}$ and $z^{\prime}$-band images for CL 0016 and RX J0153, respectively, using the SExtRactor software (Bertin and Arnouts 1996). We detected all objects with at least 8 connected pixels $(0.33$ $\operatorname{arcsec}^{2}$, equivalent to the area of the PSF) more than 2 $\sigma$ above the median sky. The images in all filters were aligned by centroiding stars throughout the field and fitting a stretch and shift. We used a fixed $2^{\prime \prime}$ diameter aperture (corresponding to $13-15 \mathrm{kpc}$ at the cluster redshifts) when measuring the galaxy colors. The magnitude MAG_AUTO was used as a measure of the total magnitude.

\subsection{Photometric Redshifts}

In order to map the structures of clusters out to lowdensity regions around the cluster cores, the key requirement is the removal of unassociated galaxies in the foreground and background that dominate at the outskirts of clusters. This is important to maximize the contrast of the member galaxies on the projected sky.

Spectroscopy is, of course, the ideal method to remove field contamination from our sample. It is not practical, however, to obtain spectroscopy for $\gg 10000$ very faint galaxies required for our analysis. We therefore exploit the photometric redshift (phot- $z$ ) technique, as an observationally efficient and reliable method to largely remove contamination ( $\sim 90 \%$ over the whole Suprime-Cam field). We applied the photometric redshift code developed by Kodama, Bell, and Bower (1999) to the galaxies in our full photometric catalog in $B V R I$ (CL 0939), $B V R i^{\prime} z^{\prime}$ (CL 0016), and $V R i^{\prime} z^{\prime}$ (RX J0153), respectively. We note that these combinations of passbands neatly cover the $4000 \AA$ break features at the rest-frame of each cluster, which is essential to obtain accurate photometric redshifts, especially for red cluster members (Kodama et al. 1999); typical uncertainties of only $|\Delta z|=0.05-0.1$ are expected. The photometric redshift technique is now widely used to trace large-scale structures in the distant Universe (e.g., Connolly et al. 1996; Kodama et al. 2001; Gray et al. 2003; Nakata et al. 2005).

In order to apply the photometric redshift technique, the colors of the model galaxies (templates) and those of the observed galaxies must match perfectly well. In re- ality, a zero-point mismatch between the observed data and the model spectra often exists. First of all, an absolute flux calibration in the observed data is difficult with only a small number of photometric standards observed during the nights (1-2 per band). Secondly, the synthesized model spectra inevitably have uncertainties on the order of 0.05-0.2 magnitudes (Charlot et al. 1996). Taking these uncertainties into account, we shift our model magnitudes by $0.00-0.15$ magnitudes so that the models can reasonably reproduce the observed colors of the red colormagnitude sequences in the cluster cores, because the redsequence colors of clusters are known to be good guides of passive evolution (e.g., Kodama et al. 1998).

The definition of our photometric members (phot- $z$ members) for CL 0939 is $0.32 \leq z \leq 0.48$ or $-0.09 \leq \Delta z \leq$ 0.07 , as used in Kodama et al. (2001). However, for the other two clusters (CL 0016 and RX J0153), we narrowed the redshift ranges to $0.50 \leq z \leq 0.58$ and $0.78 \leq z \leq 0.86$, respectively, or $-0.05 \leq \Delta z \leq 0.03$, to further suppress any remaining contamination and to achieve higher contrasts on true structures in the lower density regions located at the cluster redshifts. The depths of the phot- $z$ sliced regions are 553, 254, and $215 \mathrm{Mpc}$ in the comoving scale for CL 0939, CL 0016, and RX J0153, respectively. It should be noted that these stringent criteria for photometric members tend to lose some true cluster members due to the error in photometric redshifts, which can be larger than $|\Delta z|>0.05$ especially for blue members. When we analyze the galaxy properties in a future paper (Tanaka et al. 2005), we will adopt much broader ranges for photometric members to achieve higher completeness at the cost of higher contamination (the remaining contamination will, however, be subtracted statistically as in Kodama et al. 2001). In the current work, we did not subtract the remaining contamination, since we concentrated on the structures of the galaxy distribution, and did not primarily consider the photometric properties of the member galaxies.

A comparison of our photometric redshifts and the spectroscopic redshifts available from the literature are briefly discussed in Kodama et al. (2001) for CL 0939, and will be reported in Tanaka et al. (2005) for CL 0016 and RX J0153. In short, we found that about $70-80 \%$ of the spectroscopic members could be assigned reasonable photometric redshifts, and were hence identified as our photometric members. We also note that a spectroscopic followup of the CL 0016 and RX J0153 clusters with FOCAS on Subaru was performed in 2004 October and the data are being processed, which will confirm membership for the bright objects.

\section{Results}

We first show color-magnitude diagrams in figure 3 to demonstrate the data that we have obtained in all available passbands. We then use these diagrams to separate the red and blue cluster members. The 2-D distributions of these photometric members are shown on two scales, close-up of the central regions of the clusters (figure 4) to 


\section{Figure1.jpg}

Fig. 1. False-color image of the central $3^{\prime} \times 3^{\prime}$ region of CL $0016+1609$ constructed from our $V, R$, and $i^{\prime}$ images. North is up and east is to the left.

\section{Figure2.jpg}

Fig. 2. False-color image of the central $3^{\prime} \times 3^{\prime}$ region of RX J0152.7-1357 constructed from our $V$, $R$, and $i^{\prime}$ images. North is up and east is to the left.

trace the sub-structures in or near the cluster cores and the whole Suprime-Cam field ( $\sim 30^{\prime}$ across; figure 5$)$ to map out the large-scale structures surrounding the cores. The optical sub-structures of the clusters are compared to the X-ray maps and show similarities (figure 4). We will then try to quantify the structures of the clusters using the shapes of the 2-D iso-density contours (figure 6) and a Fourier expansion of the galaxy distribution in the tangential direction around the cluster cores (figure 9) to discuss the roundness and filamentarity of the structures. Finally, these observed structures will be compared to the model predictions from our numerical simulation $(\nu \mathrm{GC}$; Nagashima et al. 2005) which combines the $N$-body calculation concerning the development of the dark matter halos and what is commonly known as semi-analytic modelling of galaxy formation and evolution (star formation and mergers) within the halos (figures 5 and 6 ).

\subsection{Color-Magnitude Diagrams}

Color-magnitude diagrams of the galaxies within $1 \mathrm{Mpc}$ radii from the centers of the three clusters are shown in figure 3. The photometric members selected based on the photometric redshifts are indicated by circles, while the photometric non-members are indicated by crosses. As expected, the photometric members show a tight colormagnitude relations in all colors that are composed of old passively evolving galaxies (e.g., Bower et al. 1992; Ellis et al. 1997; Stanford et al. 1998; Kodama et al. 1998; Terlevich et al. 2000). The solid lines show the predicted color-magnitude relations at the cluster redshifts from Kodama et al. (1998), which are constructed so as to reproduce the color-magnitude relation of Coma elliptical galaxies (Bower et al. 1992). The zero-points of the models are corrected as in subsection 2.2. We use these diagrams to define 'red' galaxies, 'blue' galaxies, and 'red-sequence' galaxies, based on the distance from the red color-magnitude sequence (solid lines). The galaxies bracketed by the dotted lines are defined to be 'red-sequence' galaxies, which are plotted in the middlerow panels of figure 5 . The exact definitions of the redsequence galaxies are given by the following two color ranges from the red-sequence for each cluster:

\section{0939:}

$-0.20<(V-I)-[1.74-0.0547 \times(I-18.32)]<0.10$

and

$-0.10<(R-I)-[0.62-0.0174 \times(I-18.32)]<0.05$.

\section{0016:}

$-0.20<\left(V-i^{\prime}\right)-\left[2.09-0.0627 \times\left(i^{\prime}-19.16\right)\right]<0.14$

and

$-0.10<\left(R-i^{\prime}\right)-\left[0.76-0.0251 \times\left(i^{\prime}-19.16\right)\right]<0.07$. (4)

RX J0153:

$-0.20<\left(R-z^{\prime}\right)-\left[1.96-0.0575 \times\left(z^{\prime}-19.72\right)\right]<0.10(5)$

and 
$-0.10<\left(i^{\prime}-z^{\prime}\right)-\left[0.79-0.0275 \times\left(z^{\prime}-19.72\right)\right]<0.05 .(6)$

Although red-sequence galaxies are defined in the above two colors each, they also show consistently red colors and form clear sequences in the other colors as well. The 'red' galaxies and 'blue' galaxies are separated by the dashed line on the color-magnitude diagram which shows the color bracketing the rest-frame $4000 \AA$ break. The distance of the separative line from the red color-magnitude sequence corresponds to $\Delta(B-V)=-0.2$ in the rest-frame following the original Butcher and Oemler's (1984) type definition. This is transformed to each observed frame using Kodama et al.'s (1998) population synthesis model by taking into account the color evolution (Kodama, Bower 2001). The thus-defined red and blue galaxies are shown by different symbols in figures 4 and 5 .

The vertical dot-dashed lines correspond to $M^{*}+3.5$ at the cluster redshifts assuming passive evolution; hereafter, we restrict our sample to those galaxies brighter than these limits.

\subsection{Substructures in the Cluster Cores}

The 2-D distribution of the photometric members defined as in subsection 2.2 in the central regions of the clusters, are shown in the left panels of figure 4 . The substructures are clearly seen in all three clusters under study. We use the archived XMM data of these three clusters and reproduce the X-ray contour maps at $0.3-5.0 \mathrm{keV}$ in the right panels for a comparison. These data were originally presented in De Filippis, Schindler and Castillo-Morales (2003), Worral and Birkinshaw (2003), and Jones et al. (2004) for CL 0939, CL 0016 and RX J0153, respectively. The similarity of the structures in the cluster cores and their neighbouring regions between the optical galaxy distribution and the current X-ray images (hot gas trapped in the potential well) is seen as described individually, as follows:

- CL 0939: The core structure is composed of two major clumps stretching from East to West and to SW. Many filaments to North, NW, South and NE are also clearly identified in the optical image. In the X-ray images only the North and the NW extensions are visible. We also note that the similarity is also seen between the weak lensing mass map and the Rosat X-ray image (Iye et al. 2000).

- CL 0016: The galaxy distribution is rather round and featureless within the central $\sim 2^{\prime}$ radius region. Beyond this area the optical distribution tends to be stretched in the NE-SW direction which links to the distinct SW clump at $(\Delta$ R.A., $\Delta$ Dec. $)=\left(4^{\prime},-8.5^{\prime}\right)$ (§3.3; figure 5).

- RX J0153: The central three red clumps are aligned linearly in the NE-SW direction forming a chain like structure. This filament corresponds to two major clumps in the X-ray image. Also, the NW-SE filament perpendicular to the NE-SW filament crossing at the NE edge is seen both in the optical and X-ray images. It is notable that the southern extension of the filament is seen in the op- tical image, but is not clear in the X-ray image. The small clump at $(\Delta$ R.A., $\Delta$ Dec. $)=\left(4^{\prime},-0.9\right)$ has been recently confirmed to lie at the cluster redshift by Demarco et al. (2005).

\subsection{Large-Scale Structures beyond the Cores}

The galaxy distribution over the entire $30^{\prime}$ fields of the three clusters is shown in figure 5 . The photometric members are shown in the left panels separated, into red and blue galaxies. Another method that picks out the redsequence galaxies has a comparable sensitivity to the redshift $(\Delta z \sim 0.05)$ for old passively evolving populations at cluster redshift, and hence it is a simple, but powerful, technique for finding structures, such as clusters (e.g., Gladders, Yee 2000; Lubin et al., 2000; Tanaka et al. 2001; Ebeling et al. 2004). The red-sequence galaxies defined by inequalities (1)-(6) are shown in the middle panels. An example of our model cluster (the most massive halo in our simulation) is shown in the right panels for a comparison (see subsection 3.5).

The large-scale filamentary structures are clearly visible in all clusters extending out to the full SuprimeCam fields, which correspond to $\sim 15-30 \mathrm{Mpc}$ across on the comoving scale. Most of the structures found in the present study are robust because they are both seen in the phot- $z$ members and in the red sequence galaxies. Also, the comoving volume within the photometric redshift slice over the observed area is $0.84,0.86$, and 1.4 $\times 10^{5} \mathrm{Mpc}^{3}$, for CL 0939, CL 0016 and RX J0153, respectively. Given the fact that the typical comoving density of clusters/groups $\left(>10^{14} M_{\odot}\right)$ is roughly $10^{-5} \mathrm{Mpc}^{-3}$ (Nagashima et al. 2005), the expected number of clusters/groups that fall randomly within our narrow redshift slice would be around unity per field. Therefore, from a statistical point of view, most of the structures seen in the redshift-sliced maps (figure 5) are likely to be in common large structures around the main bodies of the clusters, rather than randomly falling into our phot- $z$ slices and being physically independent.

Since XMM provides a $30^{\prime}$ diameter field of view, the XMM archived data enabled us to search for any diffuse X-ray emission from the groups along the large-scale structures. However, we did not find any significant emission from the groups outside the $3^{\prime}$ radii from the cluster centers, except for a known group, RX J0018.3+1618, in CL 0016 and the south group in RX J0153 (see below). The upper limits of the X-ray luminosities of these optically identified, but X-rays undetected, groups were estimated to be 2-4, 1-5, and 10-20 in units of $10^{43} \mathrm{erg} \mathrm{s}^{-1}$ (0.5-10 keV) for CL 0939, CL 0016, and RX J0153, respectively. Please note that some groups are out of the XMM field of view, or very close to the edge, where sensitivity drops significantly.

We discuss below the large-scale structures of each cluster individually.

- CL 0939: As was reported in Kodama et al. (2001), although this cluster is dominated by a red core, many filaments coming out from the core and ex- 


\section{Figure3.jpg}

Fig. 3. Color-magnitude diagrams of galaxies within 1 Mpc radii from the cluster centers of CL 0939 (top left), CL 0016 (bottom), and RX J0153 (top right). The solid lines show the location of the red color-magnitude sequence, and the dotted lines indicate the definition of the red-sequence galaxies that should fall between these two lines. The dashed lines represent the Butcher-Oemler (1984) type definition of blue galaxies, which corresponds to $\Delta(B-V)=-0.2$ at $z=0$ with respect to the red color-magnitude sequence (see Kodama, Bower 2001). The red-sequence galaxies are shown by the red symbols. The photometric members and non-members based on photometric redshifts are shown by circles and crosses, respectively. The vertical dot-dashed lines indicate the magnitude cuts corresponding to $M^{*}+3.5$ in the case of passive evolution. Hereafter, we restrict our sample to those galaxies brighter than these limits.

\section{Figure4.jpg}

Fig. 4. Comparison of our galaxy distribution in the optical with the X-ray images for the central regions of CL $0939(z=0.41)$, CL $0016(z=0.55)$ and RX J0153 ( $z=0.83)$ (from top to bottom panels). In the left panels, we show the 2-D distribution of our cluster member candidates based on the photometric redshifts (see figure 5), and the contours show the local 2-D number density of galaxies at $1.5,2,3,4,5 \sigma$ above the mean density within the central $14^{\prime} \times 14^{\prime}$ fields. We applied Gaussian smoothing $($ sigma $=$ $0.1 \mathrm{Mpc}$ ) to each galaxy and combined the tails of the Gaussian wings to measure the local density at a given point. A bin size of $0.05 \mathrm{Mpc}$ (physical) was used to draw the iso-density contours. The coordinates are shown relative to the center of the main cluster. In the right panels, we show X-ray contour maps at $0.3-5.0 \mathrm{keV}$, reproduced from the archived XMM data. The smoothing length is $12 . " 8$, comparable to the point-spread function of XMM. The X-ray contours are logarithmically spaced by a factor of 1.5 . The bottom contours correspond to 1.4, 1.9, and $1.1 \times 10^{-6} \mathrm{cts} \mathrm{s}^{-1} \operatorname{arcsec}^{-2}$ for CL 0939, CL 0016, and RX J0153, respectively. These numbers are transformed to 3.3, 4.5 and $2.7 \times 10^{-18} \mathrm{erg} \mathrm{s}^{-1} \mathrm{~cm}^{-2} \operatorname{arcsec}^{-2}$, respectively, if the thermal emission of $5 \mathrm{keV}$ plasma is assumed. Note that some X-ray sources shown by 'P.S.' are likely to be point sources and are irrelevant in this study.

tending to large radii have also been identified. This gives us an impression that the cluster is indeed located at the node of the cosmic web, and that the surrounding material is being assembled to the cluster core along these filaments, as the numerical simulations suggest (e.g., Ghigna et al. 1998; Yahagi et al. 2004).

- CL 0016: This field is known as a triple cluster in X-rays (ROSAT) and optical observations (Connolly et al. 1996). The clumps at $(\Delta$ R.A., $\Delta$ Dec. $)=\left(0^{\prime}, 0^{\prime}\right),\left(-4^{\prime},-8.5\right)$ and $(3$. $\left.' 5,-24^{\prime}\right)$ are all X-rays detected, and their physical associations have been confirmed spectroscopically. The central clump and the south clump are named RX J0018.3+1618 (Hughes et al. 1995) and RX J0018.8+1602 (Connolly et al. 1996), respectively. We now find the structures connecting these three major clumps, composed of several new clumps, such as that at $\left(-3^{\prime},-12.5\right)$, and the large NE-SW filaments coming out from the richest cluster core. All of these structures seem to align and form a huge connected filament extending more than $20 \mathrm{Mpc}$ (comoving).

- RX J0153: This cluster has a core of very complicated shape, and multiple filaments have been identified coming out from the core, which may be connected to the small clumps scattered around the core. The SE clump at $(\Delta$ R.A., $\Delta$ Dec. $)=\left(4^{\prime},-9^{\prime}\right)$ was detected in an XMM image, but it is likely to be a foreground structure at slightly lower redshift, since the location of the red color sequence is slightly offset to bluer color [by $\Delta\left(R-z^{\prime}\right)=0.1 \mathrm{mag}$ ] in this system compared to that of the main cluster. In fact, this clump disappears in the middle panel of 


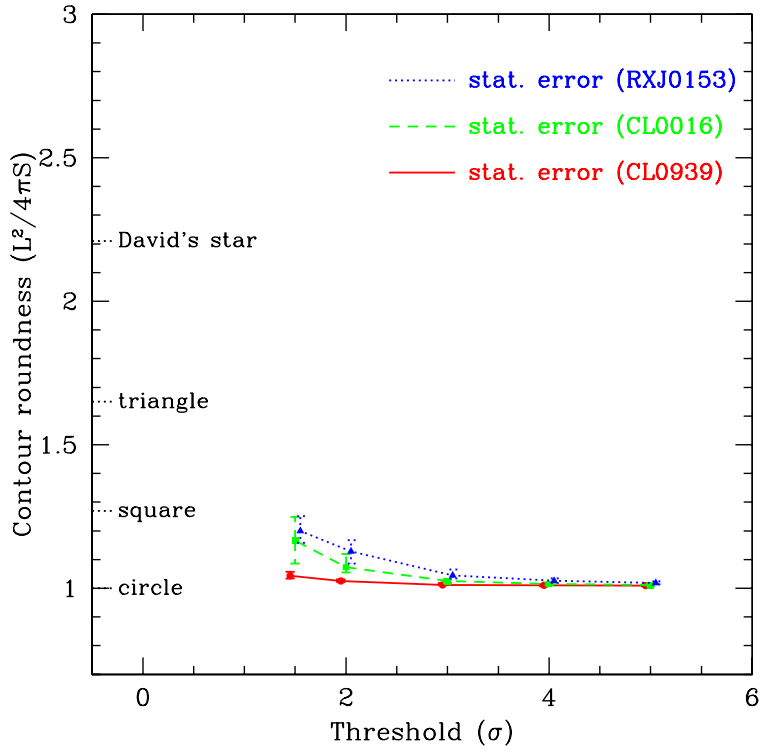

Fig. 7. Statistical errors on the roundness index of iso-density contours $\left(L^{2} / 4 \pi S\right)$ for the three clusters. These were estimated from a Monte-Carlo simulation for each cluster by generating artificial clusters that have perfectly round galaxy distribution with $\Sigma \propto r^{-2}$ within $2 \mathrm{Mpc}$ radius (flat distribution within $0.2 \mathrm{Mpc}$ ). On top of them we add field contamination randomly over the entire field. The number of the generated cluster galaxies within the $2 \mathrm{Mpc}$ radius and that of the generated field galaxies are equal to the statistically estimated numbers from the observation, assuming that a low-density field in the image corresponds to a general field. We then measured the shape indices skewed by the contamination. The data points and the associated error-bars show the median and $16 \%-84 \%$ range of the distribution of 10 Monte-Carlo runs.

figure 5 where only red-sequence galaxies consistent with the cluster redshift are plotted. In any case, this rather complicated structure in and around the core (figures 4 and 5) suggests that it is still in a dynamically young stage of cluster-scale assembly at this high redshift. This is in qualitative agreement with the build-up of clusters in the CDM simulations (e.g., Ghigna et al. 1998; Yahagi et al. 2004).

\subsection{Morphology of Clusters: Quantifying the Structures}

To trace the evolution of cluster structures with time, and to compare it with numerical simulations, we tried to quantify the structures of the phot- $z$ member distribution by defining the following two quantities: (1) roundness of the iso-density contour as a function of the threshold of the contour; (2) filamentarity of galaxy distribution in the tangential direction. We intuitively expect that at high redshifts, clusters are in the process of vigorous assembly by capturing the surrounding systems along the filaments, and thus having more and more clumpy and filamentary structures in and around the cluster cores and large deviation from a spherical distribution.

The roundness index of an iso-density contour is defined by

$$
C=L^{2} / 4 \pi S
$$

in analogy to Schmalzing et al. (1999), where $L$ is the length of the contour, and $S$ is the area surrounded by the contour. This index represents the roundness of the contour and a perfect circle gives unity. A square and an equilateral triangle give shape indices of 1.27 and 1.65, respectively. David's star, where an equilateral triangle is placed up-side down on top of another triangle, gives $C=$ 2.21. The larger is the index, the larger is the deviation from a circle.

The $C$ indices are measured for each iso-density contour of the phot- $z$ members drawn on the major clump at or near the cluster center (figure 5 ). We plot them as a function of the threshold of the contour $(\sigma)$ in the left panel of figure 6 for the three clusters at different redshifts. The general trend seen in all clusters is that the inner (higher) contours are closer to circles, but the outer (lower) contours show larger deviations from circles, indicating more complicated structures that reflect the filaments coming out from the main bodies, as is evident from the 2-D distribution in figures 4 and 5 .

The observed clusters after the photometric selection still contain non-negligible field contamination at the lower contours, and it can skew the intrinsic shape of cluster galaxy distribution. To estimate this effect, we generated a perfectly round cluster and added field contamination randomly over the entire field by a Monte-Carlo simulation. We then measured the shape indices, $C$, to see how much the round galaxy distribution was skewd. However, as shown in figure 7 , such a statistical effect is found to be small. On the other hand, if the intrinsic distribution of cluster galaxies are far from round, the uniform (random) field contamination would make the contours rounder and the measured $C$-indices would be lower limits. In any case, we conclude that the statistical error in measuring the contour shapes is not so large as to affect the trends we see in figure 6 .

CL 0939 and CL 0016 have similar shape indices, but the highest redshift cluster, RX J0153, has significantly higher values than the two lower redshift clusters at all contour levels. This might indicate a possible evolutionary effect, but we cannot make any general remarks with only three clusters and one each at each epoch. We defer any conclusion until we complete observations of most of the 15 clusters and make a statistical analysis on this plane. We compare the shape indices between the observed clusters and the simulated clusters in the right panel of figure 6 (see subsection 3.5).

In order to quantify the visual impression of filametary structures of the observed distant clusters, we also plotted the tangential distribution of galaxies (figure 8) and calculated the power of the Fourier expansion of the galaxy distribution around the averaged density in the tangential direction (figure 9), within the radial range of $0.3<R_{\mathrm{c}}<1.5 \mathrm{Mpc}$ of each cluster. The galaxies within this radial range (total number is $n$ ) are sorted in order of $\theta$, and the amplitude $(A)$ in figure 8 at the $i$-th galaxy 


\section{Figure5.jpg}

Fig. 5. Panoramic maps of CL $0939(z=0.41)$, CL $0016(z=0.55)$, and RX J0153 $(z=0.83)$ clusters (from top to bottom panels in the left and the middle rows). In the left panels, we show the photometric member candidates which were selected with redshift cuts of $0.32 \leq z \leq 0.48,0.50 \leq z \leq 0.58$ and $0.78 \leq z \leq 0.86$, respectively. The contours show the local 2-D number density of these galaxies at $1.5,2,3,4,5 \sigma$ above the mean density over the whole field. We applied Gaussian smoothing (sigma $=0.2 \mathrm{Mpc}$ ) to each galaxy and combined the tails of the Gaussian wings to measure the local density at a given point. A bin size of $0.1 \mathrm{Mpc}$ (physical) was used to draw iso-density contours. The coordinates are shown relative to the center of the main cluster. Large-scale filamentary structures $(>10 \mathrm{Mpc})$ are seen in all clusters. In the middle row, we show the red galaxies on the color-magnitude sequence of each cluster. The red galaxies were selected based on the $V R I$ colors for CL 0939, $V R i^{\prime}$ colors for CL 0016, and $R i^{\prime} z^{\prime}$ colors for RX J0153, respectively, and the color slice cuts were applied to isolate the passively evolving galaxies at cluster redshifts (see text and figure 3 for details). For a comparison, an example of the simulated clusters at $z=0.40, z=0.57$, and $z=0.82$ is shown from top to bottom in the right row, respectively (Nagashima et al. 2005). The cluster is projected over depth of $30 \mathrm{Mpc}$ (comoving), and both the red and blue members are plotted (definitions of the red and blue galaxies and the magnitude cuts are exactly the same as for the observed clusters).
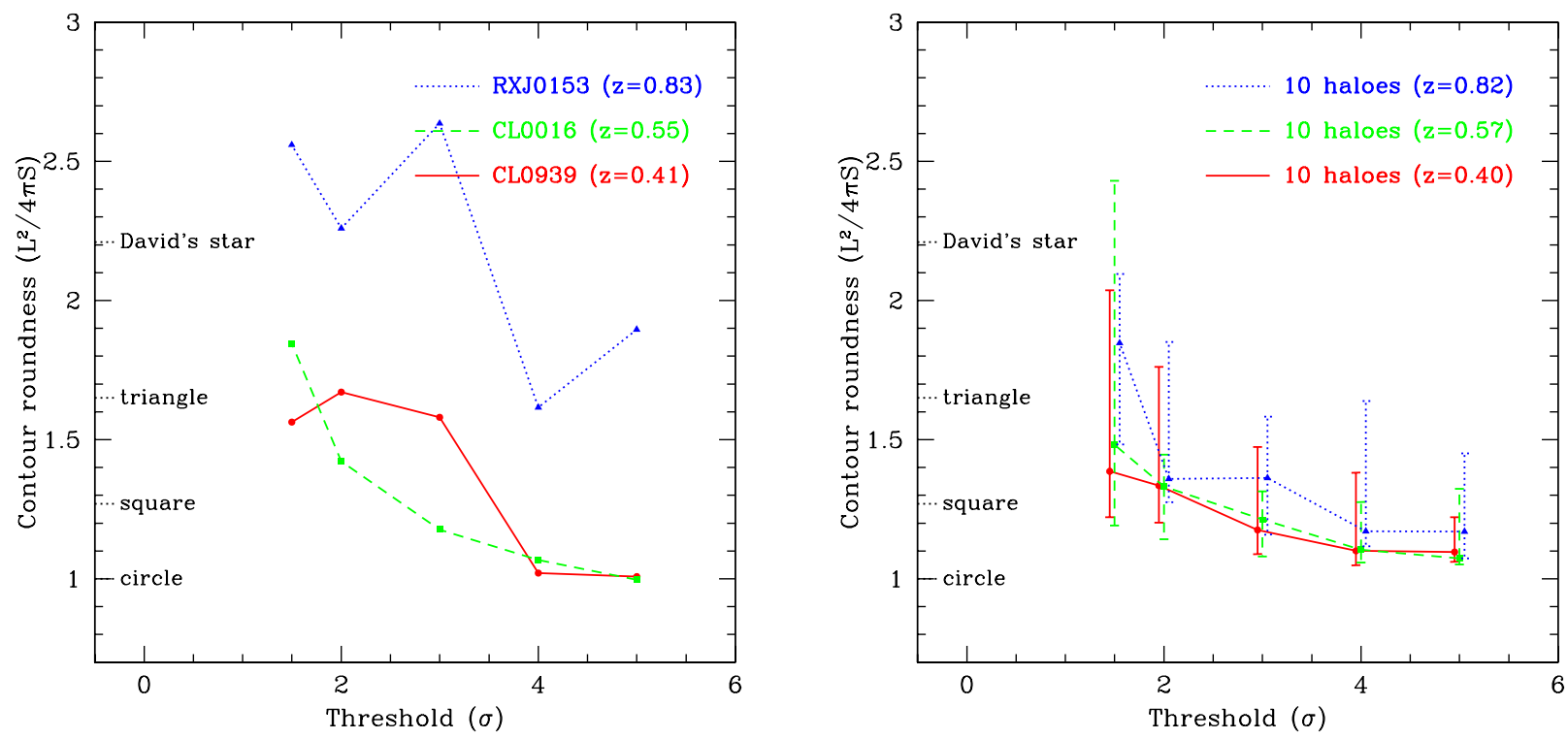

Fig. 6. Roundness of iso-density contours $\left(L^{2} / 4 \pi S\right)$ as a function of the threshold of the contours, where $L$ is the length of the contour and $S$ is the area surrounded by the contour. Only the major clump near or at the cluster center $(0,0)$ is used. Observed data are plotted in the left panel, while the model predictions are shown in the right panel. The data points and the associated error-bars in the right panel show the median and 16\%-84\% range of the distribution of 10 model halos at each threshold, respectively. 

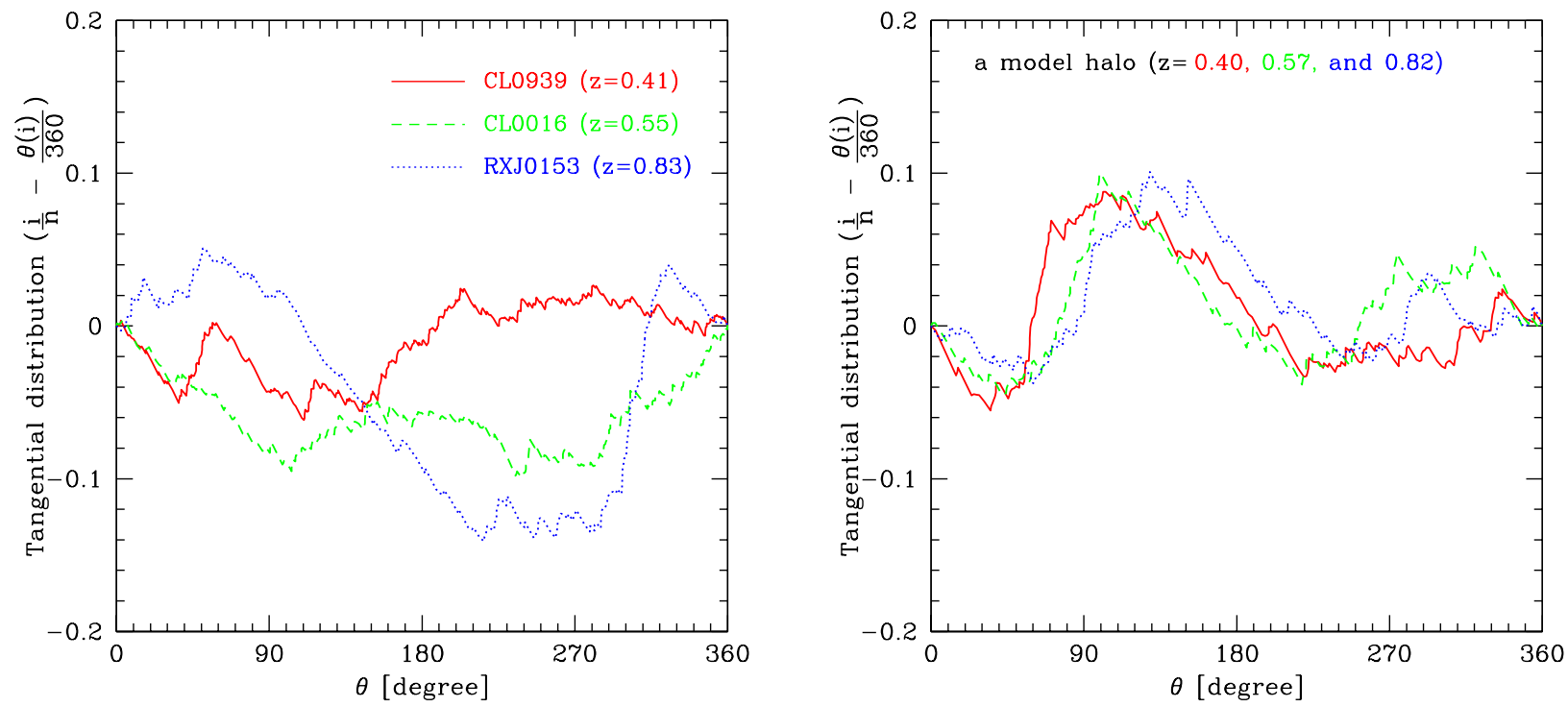

Fig. 8. Tangential galaxy distribution within the radial range of $0.3<R_{\mathrm{C}}<1.5 \mathrm{Mpc}$ centerd on the positions of $(\Delta$ R.A., $\Delta$ Dec.) $=(0 ! 4,0 ! 2),\left(0^{\prime}, 0^{\prime}\right)$, and $(0 \prime 4,0 \prime 6)$, for CL 0939, CL 0016, and RX J0153 clusters, respectively. The galaxies within the above radial range (total number is $n$ ) are sorted in order of $\theta$, and the amplitude at the $i$-th galaxy at $\theta(i)$ is given by $\frac{i}{n}-\frac{\theta(i)}{360^{\circ}}$. A straight line at zero would mean the uniform distribution of galaxies in the tangential direction (equal spacing in $\theta$ ). The decrement and increment of the curves reflect the lower and higher densities compared to the averaged density, respectively (left panel). An example of our model cluster (most massive halo in our simulation) is shown in the right panel.

at $\theta_{i}$ is defined as

$$
A\left(\theta_{i}\right)=\frac{i}{n}-\frac{\theta_{i}}{360^{\circ}}
$$

Also, the normalized Fourier power, $P(\theta)$, in figure 9 is defined as

$$
P(\theta)=\left[\frac{1}{n} \sum_{i=1}^{n} \cos k \theta_{i}\right]^{2}+\left[\frac{1}{n} \sum_{i=1}^{n} \sin k \theta_{i}\right]^{2},
$$

where $\theta=\frac{360^{\circ}}{k}(k=1,2,3, \ldots, 36)$. If the galaxies are distributed with equal spacing in the $\theta$ direction, $A=P(\theta)=0$ (constant), by definition. The results for the observed clusters are shown in the left panels, while an example of our model cluster (most massive halo in our simulation) is shown in the right panels for comparison (see subsection $3.5)$.

The tangential distribution of galaxies in the CL 0939, CL 0016 and RX J0153 clusters show high powers at $60^{\circ}$, $180^{\circ}$, and $90^{\circ}$, respectively, which reflect the visual impression of the structures of these clusters: namely, many $(\sim 6)$ filaments coming out from the core of CL 0939 (figure 5), the global linear structure in the NE-SW direction of CL 0016 (figure 4), and the ' $T$ '-shape structure in the core of RX J0153 (figure 4).

\subsection{Comparison with Numerical Simulations}

We compare our results with $\nu$ GC simulation of galaxy formation (Nagashima et al. 2005) which is based on the CDM based $N$-body simulations (Yahagi et al. 2004) and on the Mitaka semi-analytic model of galaxy formation
(Nagashima, Yoshii 2004). The simulation volume was a $100 \times 100 \times 100 \mathrm{Mpc}^{3}$ cube (comoving), and the number of particles used was $512^{3}$. The mass of each particle was $3.04 \times 10^{8} \mathrm{M}_{\odot}$. The adopted cosmological parameters were $\left(h_{70}, \Omega_{m}, \Omega_{\Lambda}\right)=(1.0,0.3,0.7), \Omega_{\mathrm{b}}=0.04$, and $\sigma_{8}=1$. The power spectrum given by Bardeen et al. (1986) was used. The model successfully reproduced many observational quantities, such as the luminosity functions, gas fractions, and faint galaxy counts (Nagashima et al. 2005).

Halos were identified from the simulation data by applying the friends-of-friends algorithm with a linking length of $20 \%$ of a mean separation of particles at each redshift. We selected those halos that contain equal to, or more than 10 particles; hence, the mass resolution was $3 \times 10^{9} M_{\odot}$. The masses of the halos were determined at each epoch within the iso-density surface at which the mass density was 200 times higher than the mean density of the Universe. We used 10 of the most massive halos at $z=0.41,0.57$, and 0.82, respectively, for comparing with CL 0939, CL 0016 and RX J0153. The masses of the 10 selected cluster halos ranges from $5.5 \times 10^{14} M_{\odot}$ (heaviest) to $9.5 \times 10^{13} M_{\odot}$ (lightest) at $z=0.82$, and these masses evolved by a factor of $1.3-2$ by the present-day, except for the halos incorporated into more massive halos. The masses of the observed three clusters inside the $1 \mathrm{Mpc}$ radii are in the range of $2-5 \times 10^{14} M_{\odot}$ (De Filippis et al. 2003; Worrall, Birkinshaw 2003; Huo et al. 2003), spanning the range of the model cluster masses. We note that the mass range of the model halos extends to slightly smaller mass compared to that of the observed clusters due to the limitation 

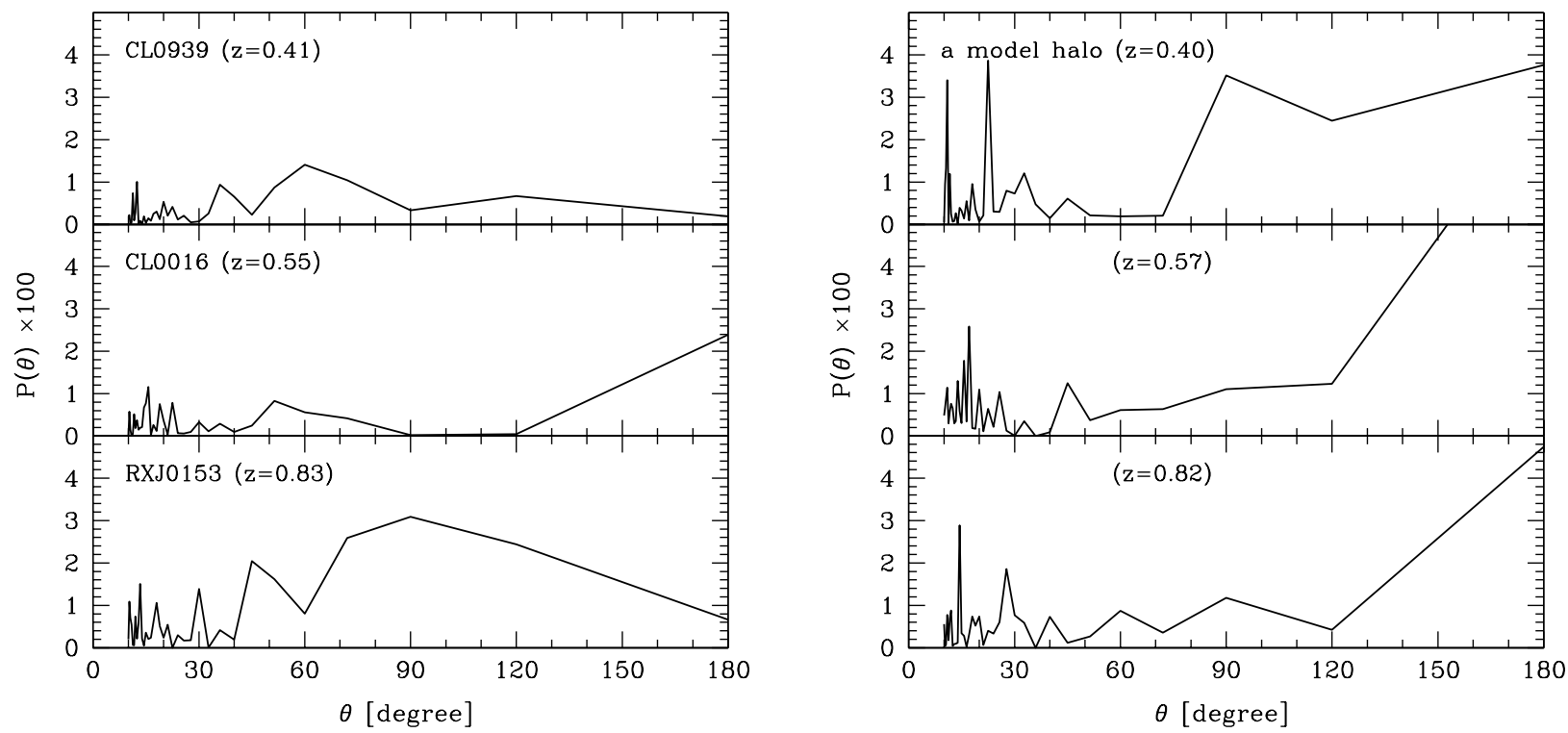

Fig. 9. Normalized power of the Fourier expansion of the galaxy distribution around the averaged density in the tangential direction, within the radial range of $0.3<R_{\mathrm{C}}<1.5 \mathrm{Mpc}$ centerd on the positions of $(\Delta$ R.A., $\Delta$ Dec. $)=\left(0{ }^{\prime} 4,0^{\prime} \cdot 2\right),\left(0^{\prime}, 0^{\prime}\right)$, and $\left(0{ }^{\prime} 4,00^{\prime} 6\right)$, for CL 0939, CL 0016, and RX J0153 clusters, respectively (left panel). An example of our model cluster (most massive halo in our simulation) is shown in the right panel.

of the simulation box. Therefore, we may need a larger simulation to make a truly fair comparison. The right panels in figures 5,8 , and 9 show the 2-D projection of the most massive halo in the simulation box, as well as its tangential distribution and its Fourier expansion, respectively. The filamentary and clumpy structures of the model clusters look very similar to those of the observed clusters.

In order to make a quantitative comparison, the contour shapes, $C$, were calculated for these 10 model clusters at each epoch $(z=0.40,0.57$, and 0.82$)$, exactly in the same manner as for the observed clusters, where we used a smoothing length of $0.2 \mathrm{Mpc}$ and bin size of $0.1 \mathrm{Mpc}$ in the physical (proper) scale. The model clusters were projected to 2-D in this calculation over a depth of $30 \mathrm{Mpc}$ (comoving). The shape indices of the model clusters are shown in the right panel of figure 6 . The error-bars show the 16\%-84\% range of the distribution of 10 halos at each threshold. The model values are in good agreement with the observed ones for $z \sim 0.4$ and $z \sim 0.55$, and the general trend that the contour shapes deviate from circles with decreasing density is seen in the model clusters as well. The higher redshift clusters at $z=0.82$ in the model, however, show much lower shape indices than observed, although they are still systematically slightly larger than those of the lower redshift clusters in the model. The evolution of the contour shapes seems to be weaker in the model than observed, although the number of observed data points should be increased to properly discuss the evolution.

\section{Summary}

We have started a distant cluster project, PISCES, on Subaru. Its general concepts and first results concerning the cluster structures of three clusters are presented in this paper. We have found spectacular large-scale structures across $\sim 30^{\prime}$ scales around the well-studied cluster cores of the three clusters, i.e., CL $0939(z=0.41)$, CL 0016 $(z=0.55)$, and RX J0153 $(z=0.83)$ based on multicolor wide-field imaging with Suprime-Cam on Subaru. These structures are characterized by filaments coming out from the core and bridging some clumps along the filaments. These overall large-scale structures are in qualitative agreement with the numerical simulations, which predict an infall of clumps into the cluster cores travelling along the filaments during the course of cluster-scale assembly.

Moreover, we have taken a closer look at near or inside the cluster cores, and have identified complicated substructures that seem to be connected to the outer structures, and hence suggesting a recent accretion of matter from the surrounding filaments.

The substructures of the main clusters were compared with the numerical simulation $(N$-body + semi-analytic model) applying the deviation of shapes of the iso-density contours from perfect circles. A quantitative agreement between the observation and the model was found at $z \sim 0.4$ and $z \sim 0.55$, although the model predicts less evolution at $z \sim 0.83$. We do not know, however, whether this is a true evolutionary effect, or just an exception. To increase the sample of clusters is obviously needed to quantify any evolutionary signatures of clusters by distinguishing from the individualities of clusters. Also, we need 
to extend the simulation box while keeping its resolution to have a statistically fair sample of rich clusters, so that we can directly compare them with our observed clusters.

The optical substructures in the inner regions of clusters and their neighbouring regions were also compared to the X-ray maps reproduced from the archived XMM data, and were found to have a good correlation with the Xray extended emissions. This indicates that most of these sub-systems near the cluster cores are physically bound objects, rather than chance projections along the lines of sight. We are therefore likely to be witnessing a hierarchical growth of clusters.

We acknowledge Drs. Richard Bower, Laurence Jones and John Blakeslee for helpful discussions. We also thank Drs. K. Saigo and K. Ichiki at NAOJ for helping us to develop the software used in the analysis. This work is financially supported in part by a Grant-in-Aid for the Scientific Research (No. 15740126, 14102004, 16340053, and 16540223) by the Ministry of Education, Culture, Sports, Science and Technology. M.T., N.M., and T.O. acknowledge the JSPS research fellowship.

\section{References}

Adabi, M. G., Bower, R. G., \& Navarro, J. F. 2000, MNRAS, 314,759

Ajiki, M., et al. 2003, AJ, 126, 2091

Bardeen, J. M., Bond, J. R., Kaiser, N., \& Szalay, A. S. 1986, ApJ, 304, 15

Bertin, E., \& Arnouts, S. 1996, A\&AS, 117, 393

Bower, R. G., Lucey, J. R., \& Ellis, R. S. 1992, MNRAS, 254, 601

Butcher, H., \& Oemler, A. Jr. 1984, ApJ, 285, 426

Cen, R., \& Ostriker, J. P. 1993, ApJ, 417, 415

Charlot, S., Worthey, G., \& Bressan, A. 1996, ApJ, 457, 625

Connolly, A. J., Szalay, A. S., Koo, D., Romer, A. K., Holden, B., Nichol, R. C., \& Miyaji, T. 1996, ApJ, 473, L67

De Filippis, E., Schindler, S., \& Castillo-Morales, A. 2003, A\&A, 404, 63

Demarco, R., et al. 2005, A\&A, in press (astro-ph/0411386)

Donahue, M., \& Stocke, J. T. 1995, ApJ, 449, 554

Dressler, A. 1980, ApJS, 424, 565

Dressler, A., et al. 1997, ApJ, 490, 577

Ebeling, H., Barrett, E., \& Donovan, D. 2004, ApJ, 609, L49

Ellis, R. S., Smail, I., Dressler, A., Couch, W. J., Oemler, A. Jr, Butcher, H., \& Sharples, R. M. 1997, ApJ, 483, 582

Fukugita, M., Shimasaku, K., \& Ichikawa, T. 1995, PASP, 107, 945

Ghigna, S., Moore, B., Governato, F., Lake, G., Quinn, T., \& Stadel, J. 1998, MNRAS, 300, 146

Gladders, M. D., \& Yee, H. K. C. 2000, AJ, 120, 2148

Gray, M. E., Wolf, C., Meisenheimer, K., Taylor, A., Dye, S., Borch, A., \& Kleinheinrich, M. 2004, MNRAS, 347, L73

Grego, L., Carlstrom, J. E., Reese, E. D., Holder, G. P., Holzapfel, W. L., Joy, M. K., Mohr, J. J., \& Patel, S. 2001, ApJ, 552, 2

Gunn, J. E., \& Stryker, L. L. 1983, ApJS, 52, 121

Hashimoto, Y., \& Oemler, A. J. 2000, ApJ, 530, 652

Hasinger, G., et al. 1998, A\&A, 340, L27

Henry, J. P. 2000, ApJ, 534, 565

Henry, J. P., et al. 1997, AJ, 114, 1293
Hughes, J. P., Birkinshaw, M., \& Huchra, J. P. 1995, ApJ, 448, L93

Huo, Z.-Y., Xue, S.-J., Xu, H., Squires, G., \& Rosati, P. 2004, AJ, 127, 1263

Iye, M., et al. 2000, PASJ, 52, 9

Iye, M., et al. 2004, PASJ, 56, 381

Jeltema, T. E., Canizares, C. R., Bautz, M. W., Malm, M. R., Donahue, M., \& Garmire, G. P. 2001, ApJ, 562, 124

Jones, L. R., et al. 2004, in Cluster of Galaxies: Probe of Cosmological Structure and Galaxy Evolution, ed J. S. Mulchaey, A. Dressler, \& A. Oemler (Cambridge: Cambridge University Press), 25

Joy, M., et al. 2001, ApJ, 551, L1

Kaiser, N. 1984, ApJ, 284, L9

Kitayama, T., Sasaki, S., \& Suto, Y. 1998, PASJ, 50 ,1

Kodama, T., Arimoto, N., Barger, A. J., \& Aragón-Salamanca, A. 1998, A\&A, 334, 99

Kodama, T., Balogh, M. L., Smail, I., Bower, R. G., \& Nakata, F. 2004, MNRAS, 354, 1103

Kodama, T., Bell, E. F., \& Bower, R. G. 1999, MNRAS, 302, 152

Kodama, T., \& Bower, R. G. 2001, MNRAS, 321, 18

Kodama, T., Smail, I., Nakata, F., Okamura, S., \& Bower, R. G. 2001, ApJ, 562, L9

Landolt, A. U. 1992, AJ, 104, 340

Larson, R. B., Tinsley, B. M., \& Caldwell, C. N. 1980, ApJ, 237,692

Lavery, R. J., \& Henry, J. P. 1986, ApJ, 304, L5

Lubin, L. M., Brunner, R., Metzger, M. R., Postman, M., \& Oke, J. B. 2000, ApJ, 531, L5

Lubin, L. M., Mulchaey, J. S., \& Postman, M. 2004, ApJ, 601, L9

Maughan, B. J., Jones, L. R., Ebeling, H., Perlman, E., Rosati, P., Frye, C., \& Mullis, C. R. 2003, ApJ, 587, 589

Maughan, B. J., Jones, L. R., Ebeling, H., \& Scharf, C. 2004, MNRAS, 351, 1193

Miyazaki, S., et al. 2002, PASJ, 54, 833

Moore, B., Katz, N., Lake, G., Dressler, A., \& Oemler, A. Jr. 1996, Nature, 379, 613

Murakami, H. 2005, Proc. SPIE, 5487, in press

Nakata, F., et al. 2005, MNRAS, 357, 1357

Nagashima, M., Yoshii, Y. 2004, ApJ, 610, 23

Nagashima, M., Yahagi, H., Enoki, M., Yoshii, Y., \& Gouda, N. 2005, submitted

Ota, N., Pointecouteau E., Hattori M., \& Mitsuda K. 2004, ApJ, 601, 120

Pointecouteau, E., Hattori, M., Neumann, D., Komatsu, E., Matsuo, H., Kuno, N., \& Böhringer, H. 2002, A\&A, 387, 56

Rosati, P., et al. 2004, AJ, 127, 230

Sato, J., Umetsu, K, Futamase, T., \& Yamada, T., 2003 ApJ, $582, \mathrm{~L} 67$

Schlegel, D. J., Finkbeiner, D. P., \& Davis, M. 1998, ApJ, 500, 525

Schmalzing, J., Buchert, T., Melott, A. L., Sahni, V., Sathyaprakash, B. S., \& Shandarin, S. F. 1999, ApJ, 526, 568

Shibai, H. 2003, Proc. of SPIE, 4850, 162

Spergel, D. N., et al. 2003, ApJS, 148, 175

Stanford, S. A., Eisenhardt, P. R. M., \& Dickinson, M., 1998 ApJ, 492, 461

Stanford, S. A., Holden, B., Rosati, P., Tozzi, P., Borgani, S., Eisenhardt, P. R., \& Spinrad, H. 2001, ApJ, 552, 504 
Stanford, S. A., Holden, B., Rosati, P., Eisenhardt, P. R., Stern, D., Squires, G., \& Spinrad, H. 2002, AJ, 123, 619

Tanaka, I., Yamada, T., Turner, E. L., \& Suto, Y. 2001, ApJ, 547,521

Tanaka, M., Kodama, T., Umetsu, K., Arimoto, N., Okamura, S., Shimasaku, K., \& Yamada, T. 2005, submitted

Terlevich, A. I., Caldwell, N., \& Bower, R. G. 2001, MNRAS, 326,1547

West, M. J., Dekel, A., \& Oemler, A. J. 1987, ApJ, 316, 1

Worrall, D. M., \& Birkinshaw, M. 2003, MNRAS, 340, 1261

Yagi, M., Kashikawa, N., Sekiguchi, M., Doi, M., Yasuda, N., Shimasaku, K., \& Okamura, S. 2002, AJ, 123, 66

Yahagi, H., Nagashima, M., \& Yoshii, Y. 2004, ApJ, 605, 709

York, D. G., et al. 2000, AJ, 120, 1579 
This figure "Figure1.jpg" is available in "jpg" format from: http://arxiv.org/ps/astro-ph/0502444v2 
This figure "Figure2.jpg" is available in "jpg" format from: http://arxiv.org/ps/astro-ph/0502444v2 
This figure "Figure3.jpg" is available in "jpg" format from: http://arxiv.org/ps/astro-ph/0502444v2 
This figure "Figure4.jpg" is available in "jpg" format from: http://arxiv.org/ps/astro-ph/0502444v2 
This figure "Figure5.jpg" is available in "jpg" format from: http://arxiv.org/ps/astro-ph/0502444v2 